\title{
Âge de retour et retour d'âge : l'asymétrie entre les sexes dans les discours médicaux en France (1770-1836)
}

The " change " and the ages of life: the asymmetry between the sexes in medical discourse surrounding the menopause in France (1770-1836)

\section{Christine Théré}

\section{OpenEdition Journals}

\section{Édition électronique}

URL : http://journals.openedition.org/clio/12740

DOI : $10.4000 /$ clio. 12740

ISSN : $1777-5299$

\section{Éditeur}

Belin

Édition imprimée

Date de publication : 1 décembre 2015

Pagination : 53-77

ISBN : 9782701194325

ISSN : $1252-7017$

\section{Référence électronique}

Christine Théré, «Âge de retour et retour d'âge : l'asymétrie entre les sexes dans les discours médicaux en France (1770-1836) », Clio. Femmes, Genre, Histoire [En ligne], 42 | 2015, mis en ligne le 01 décembre 2018, consulté le 14 mai 2020. URL : http://journals.openedition.org/clio/12740 ; DOI : https://doi.org/10.4000/clio.12740 


\section{Âge de retour et retour d'âge : l'asymétrie entre les sexes dans les discours médicaux en France (1770-1836)}

Christine THÉRÉ

La notion d'âge critique est prégnante dans les premiers calculs sur la

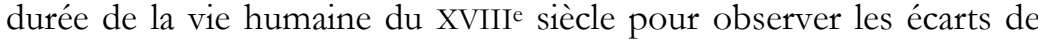
longévité entre les deux sexes, et faire le constat de la plus grande longévité des femmes. Les âges regardés comme "critiques » sont ceux qui correspondent à des «révolutions» physiologiques, la puberté qui touche les deux sexes, et la «cessation des règles » propre aux femmes. Le terme "critique», dans le vocabulaire médical, renvoie à la notion de crise dans le déroulement d'une maladie. Les expressions "âge critique» et «temps critique», qui ont un sens générique, vont prendre au fil du temps un sens secondaire pour désigner la fin des capacités reproductives chez la femme. La ménopause apparaît dès lors comme un «symptôme physiologique » de l'âge, source d'une asymétrie entre les deux sexes, renforcée par une pathologisation croissante de la «cessation des règles» à la charnière des deux siècles.

La construction médicale de la ménopause dans la France moderne et contemporaine est un objet historiographique depuis une trentaine d'années, comme en témoignent les travaux de J. Livi (1984), H. Rozembaum et J.-J. Peumery (1990), A. Tillier (2005), D. Delanoë (2006), F. Liotard-Schneider (2010) et C. McClive (2010). Ces travaux font ressortir la spécificité du cas français, et la genèse de cette histoire - des années 1760 aux années 1830 - mérite aujourd'hui d'être approfondie. Emprunter à l'histoire sociale des savoirs permet de remettre en contexte les premières productions d'une littérature médicale spécialisée qui ont déjà retenu l'attention des historiens. Cela 
conduit en premier lieu à revenir sur la variété des termes employés pour désigner la «cessation des règles» et leurs origines. Ces évolutions doivent être examinées en regard des nouvelles échelles de la vie humaine élaborées au cours de la période. Est-ce qu'une sexuation des âges de la vie, absente jusque-là, y transparaît? L'expression "âge de retour » désigne-t-elle alors un âge propre aux femmes comme le laissent penser certains auteurs ?

La pathologisation de la ménopause peut ainsi être remise dans la perspective d'un intérêt inédit pour une période transitoire de la vie qui vient repousser l'entrée dans la vieillesse proprement dite. Cet article propose d'étudier de plus près les temps forts et les principaux jalons de sa construction au cours de la période considérée, en particulier en recherchant l'écho rencontré par les travaux spécialisés dans le corps médical. Enfin, un dernier volet questionne l'existence d'un discours sur l'âge de retour au masculin et analyse la fabrique des asymétries sexuées dans les dispositifs hygiénistes destinés aux quarantenaires et aux cinquantenaires des deux sexes.

\section{Une révolution (physiologique) féminine en quête de nom}

L’inventeur du mot «ménopause » - «ménespausie » à l'origine -, Gardanne, arguait en 1812 de la confusion régnant dans le vocabulaire de ses futurs confrères - et de leurs patientes - pour justifier la création d'un nouveau terme :

Ce qui m’a engagé à donner un mot tiré du grec et de le conserver dans le cours de cet ouvrage, c'est le désir de fixer par une seule dénomination plus analogue au génie de l'art, un grand nombre de termes la plupart insignifians. Que veulent dire en effet ces mots temps critique, époque critique, âge critique, âge de retour, retour d'âge, déclin de l'âge, verte vieillesse, enfer des femmes, hiver des femmes, mort du sexe, etc. ? Ne se perd-on pas dans ce dédale de nom ?1

Ce champ lexical de la ménopause mêle à dessein des dénominations génériques alors asexuées et des locutions qui ont, elles, un contenu signifiant attesté à l'époque. Il englobe aussi des

1 Gardanne 1816 : VI. Il le transforme en ménopause dans la 2e édition (Gardanne 1821). D. Delanoë avait souligné l'intérêt de se pencher sur l'histoire du vocabulaire (2006: 19-22). 
expressions issues de registres fort différents pour plaider la nécessité d'adopter un mot savant. Les trois dernières, "enfer des femmes, hiver des femmes, mort du sexe », même si elles apparaissent dans la littérature médicale, relèvent avant tout d'une rhétorique de dramatisation de la cessation du flux menstruel et d'amplification de la perte des attributs féminins. Ces figures de style n'ont pas vocation à se substituer aux autres dénominations dans les discours savants. "Âge de retour, retour d'âge, déclin de l'âge, verte vieillesse » appartiennent, elles, à la terminologie alors en usage pour désigner les époques successives de la vie humaine. Elles font référence à des âges communs aux deux sexes, et non à un âge propre aux femmes. Gardanne ne peut l'ignorer. Cette profusion d'expressions est relativement récente dans le temps. Elle reflète l'intérêt croissant pour des âges transitoires et la sophistication des divisions de la vie à l'œuvre depuis la seconde moitié du XVIII siècle.

La médecine néo-hippocratique des Lumières reconnaît deux grands systèmes de division hérités de l'Antiquité ${ }^{2}$ : les quatre âges de la vie (enfance, jeunesse, virilité, vieillesse) et les sept âges, ou système hebdomadaire, qui repose sur une succession de périodes d'égale durée, les septénaires. Ces échelles hebdomadaires conservent parfois l'empreinte d'une autre tradition, à la fois savante et populaire, les âges ou années climatériques, désormais regardée comme une croyance superstitieuse $^{3}$. Chaque âge a ses maladies particulières et ses traitements spécifiques. Dans l'Encyclopédie de Diderot et D'Alembert, Vandenesse en distinguait sept, sans toujours en préciser les limites chronologiques. Seuls les derniers nous importent ici. La sortie de l'âge viril intervenait à 45-50 ans vers la vieillesse, subdivisée en «vieillesse proprement dite, en caducité \& décrépitude ${ }^{4}$. L'encyclopédiste situe ainsi cette dernière relativement tôt, même s'il la décompose en trois stades successifs. Un consensus naît dans les années suivantes pour la repousser dans le temps et Daignan en fixe

2 Stewart $2010: 33-68$; Beaugrand 1865.

3 Parmi les «âges climatériques », 49 et 63 ans sont réputés les plus dangereux, voir Le Bras 2000. L'usage de climatère, issu de la même racine, pour désigner la ménopause est tardif en français, Delanoë $2006: 20$.

4 Vandenesse 1751 : 170. 
ainsi les «commencemens» entre 57 ans et 63 ans, le précédent septénaire (50 ans à 56 ans) étant nommé « déclin de l'âge »- une des expressions citée par Gardanne 5 .

Les médecins naturalistes jouent alors un rôle essentiel dans la construction de divisions qui vont faire autorité jusqu'à la fin du siècle suivant. L'enjeu est de mieux distinguer des degrés dans l'enfance et la vieillesse et d'améliorer la concordance avec les trois temps de l'histoire naturelle de l'homme définis en regard de l'individu (accroissement, « état», dépérissement) et de l'espèce (les trois périodes de la vie reproductive). Collaborateur de Buffon, Louis-Jean-Marie Daubenton (1716-1799) est à l'origine de la dénomination «âge de retour» et un des premiers à mettre l'accent sur un âge mal identifié jusque-là, en lien avec la notion de "retour de l'âge ». Ces développements sur les âges de la vie sont rédigés pour l'introduction de l'Histoire naturelle des animaux de l'Encyclopédie méthodique parue en 1782. Les premiers âges constituent des étapes vers l'état de perfection, que les femmes atteignent avant les hommes, leur puberté étant plus précoce. Les bornes en sont donc relativement mobiles. L'âge viril est fixé depuis 30-35 ans jusqu'à 40-45 ans : le corps humain conserve sa perfection, même si l'apparition de la graisse en modifie déjà les proportions. L'âge de retour lui succède et Daubenton l'introduit, non sans souligner les problèmes que soulève sa dénomination :

Les Physiologistes donnent le nom de vieillesse au temps de la vie qui commence après l'âge viril, \& qui ne finit qu'à la mort : il est vrai qu'ils distinguent la verte vieillesse, senium crudum, de la vieillesse décrépite. Mais le mot de vieillesse ne peut avoir dans notre langue une signification aussi étendue. Un homme de quarante ou quarante-cinq ans n'est pas un vieillard; quoiqu'à cet âge le corps donne déjà des signes de dépérissement, ce n'est pas encore l'âge de la vieillesse: je crois qu'il serait mieux nommé l'âge de retour, puisqu'alors la nature commence à rétrograder; l'embonpoint diminue \& les fonctions de quelques parties du corps s'affoiblissent. L'âge de retour s'étend depuis quarante ou quarante-cinq ans, jusqu'à soixante ou soixante-cinq ans ${ }^{6}$.

5 Daignan 1786; sur le seuil de la vieillesse, Troyansky 1989, Bourdelais 1993, Stewart 2010.

6 Daubenton 1782: LXXIV ; sur la pauvreté du français en regard du latin, Ariès $1973: 42-43$. 
Alors que la puberté dessinait une évolution décalée entre garçons et filles, l'âge de retour n'est pas associé à la cessation des règles qui affecte le sexe féminin. Le "retour de l'âge » est un phénomène commun aux deux sexes qui se traduit selon Daubenton par l'apparition des rides et une altération de la vue. Son signe le plus marquant, en termes de santé, est l'affaiblissement de l'estomac et Daubenton rédige peu après un Mémoire sur les indigestions qui commencent à être plus fréquentes, pour la plupart des hommes, à l'âge de quarante ou quarante-cinq ans, qui rencontre un large écho, contribuant ainsi à rendre familier l'âge de retour et ses manifestations ${ }^{7}$.

La division de Daubenton est ensuite raffinée par un autre médecin naturaliste, Jean-Noël Hallé (1754-1822), toujours dans l'Encyclopédie méthodique, mais dans la partie Médecine, plus enclin à conserver une terminologie traditionnelle. Sa classification se caractérise par une extension de l'âge viril qui commence à 25 ans environ et s'achève entre 60 ans et 63 ans. Il le subdivise en virilité commençante, confirmée et décroissante, la virilité décroissante correspondant, note-til, à l'âge de retour de son confrère Daubenton, et il précise alors :

C'est à cet âge aussi que les femmes, cessant de pouvoir être mères, conservent cependant encore de la force et de la vigueur, quand elles ont passé les orages qui menacent leur sexe. Les hommes possèdent encore la faculté d'engendrer, il est vrai ; mais leurs besoins diminuent et leur ardeur s'éteint sensiblement ${ }^{8}$.

Hallé amorce ainsi une sexuation de l'âge de retour que JeanClaude de La Métherie (1743-1817) poursuit quinze ans après dans une échelle de dix périodes de 7 ans (non dénommées), intégrant des considérations sur la mortalité, et dont nous reproduisons ici les plus significatives :

[De 43 à 49 ans :] Âge critique des femmes : il en périt beaucoup. Le corps de l'homme se surcharge de plus en plus de graisse : il en périt beaucoup.

[De 50 à 56 ans :] Commence la vieillesse chez les femmes. Dernière période de la virilité chez l'homme.

7 Le mémoire, imprimé en 1785, fait l'objet d'extraits dans plusieurs journaux (Daubenton an VI).

$8 \quad$ Hallé $1787: 360$. 
[De 57 à 63 ans :] Commencement de la vieillesse de l'homme : il en périt beaucoup; on suppose qu'à 63 ans l'homme ne peut plus engendrer, ce qui n'est pas exact; mais l'homme à cet âge, comme l'animal arrivé à la vieillesse, ne donne le jour qu'à des êtres foibles. ${ }^{9}$

La Métherie traduit synthétiquement une inégalité entre les deux sexes face à la vieillesse qui est de plus en plus affirmée et admise comme ayant force de loi naturelle. Les traités voués exclusivement à l'histoire naturelle des femmes, fidèles à l'héritage de Roussel, s'accordent en général sur la précocité de son basculement dans le dernier âge de la vie. L'historiographie a déjà souligné l'importance du courant naturaliste dans la construction de ces asymétries ${ }^{10}$. La contribution d'une spécialité médicale, alors en pleine mutation, la médecine légale, est en revanche méconnue alors qu'elle constitue pourtant une source notable dans la production d'une dimension sexuée des âges de la vie, comme en témoignent les travaux de François-Emmanuel Fodéré (1764-1835). Le médecin savoyard, venu à Paris sous la Révolution, participe activement à sa transformation en une véritable «science» moderne ${ }^{11}$. L'âge est une question essentielle pour le médecin légiste et il met l'accent sur la « différence tranchante » entre les deux sexes, perceptible dès l'enfance.

Passé 25 ans, l'âge des individus devient plus difficile à déterminer, selon Fodéré, surtout pour les femmes «qui, par leur destinée particulière, ont acquis en général leur degré de consistance dès qu'elles peuvent devenir mères, et $[\ldots]$ ne sont plus susceptibles, jusqu'à l'âge de cinquante ans, des gradations qui s'observent dans le sexe masculin ${ }^{12}$. Ces gradations signalent qu'une «révolution» s'opère chez l'homme entre 35 ans et 40 ans, venant marquer le terme de la jeunesse. Fodéré s'attache ensuite à la vieillesse dont le Code pénal fixe le seuil à 70 ans. Mais à l'égard des femmes, «l'époque de la

9 La Métherie 1804 : t. II, 477-478. Comme Daubenton et Hallé, La Métherie est médecin et naturaliste.

10 Roussel 1775, six rééditions jusqu'en 1845 ; sur son héritage, Hoffmann 1995 [1977]. Parmi les contemporains de La Métherie, citons Julien-Joseph Virey (1775-1846) dont Y. Knibiehler et C. Marand-Fouquet (1983) ont montré l'importance ; voir aussi Tillier 2005 : 3-4.

11 Sur la médecine légale de l'époque, Porret $2010: 10-15$.

12 Fodéré 1813 : t. 1, 23-24. 
vieillesse légale est beaucoup plus avancée [c'est-à-dire précoce]; on les juge vieilles à l'âge de quarante-cinq à cinquante ans » et effectivement l'âge de 45 ans est pris en compte pour le divorce par consentement mutuel, non admis si l'épouse l'a atteint ${ }^{13}$. Cette vieillesse légale, relative à la fécondation, fausse à ses yeux la perception de la femme âgée : en réalité, elle n'est pas plus vieille que l'homme, "absolument parlant», et paraît même "souvent se conserver plus longtems $\gg^{14}$. Le médecin légiste relativise ainsi l'incidence de la ménopause, et renverse même l'inégalité entre les sexes face aux premiers signes de vieillissement.

Pour désigner les troubles de la ménopause, Fodéré parle des « incommodités qui naissent de l'âge critique ». Gardanne, rappelons-le, recensait aussi les locutions voisines, "temps critique » et "époque critique ». Les trois expressions se concurrencent et, contrairement à la dénomination "âge de retour», elles ont une origine plus diffuse. Chacune d'entre elles conservent un emploi générique pour qualifier des étapes estimées dangereuses, tant au plan moral qu'au physique, de l'existence humaine, en premier lieu la puberté et la jeunesse. L'usage de «temps critique », en ce sens restreint, est recensé pour la première fois dans le Dictionnaire de l'Académie française dans son édition de 1798, à l'article «Critique ». L'Encyclopédie méthodique confirme l'antériorité de cette expression sur les autres, son succès est toutefois éphémère ${ }^{15}$. Rappelons qu'à l'époque «temps critique » signifie aussi la période menstruelle et le retour du flux périodique. Dans l'édition suivante du Dictionnaire de l'Académie française (1835), l'article « Critique » indique à la fois « temps critique » et « âge critique » pour signifier «celui où une femme cesse d'avoir ses règles ». "Âge critique » est devenu le plus commun et fait désormais l'objet d'une sous-entrée à "Âge », absente dans les précédentes éditions. C'est la dénomination que Gardanne conserve au moment de publier sa thèse, en dépit du nouveau mot qu'il a forgé.

Jusqu'aux années 1760 les médecins s'en tenaient le plus souvent aux formules qui décrivent le phénomène biologique - cessation

13 Recueil des lois ... an XI : 523 (Loi du 30 ventose an XI).

14 Fodéré 1813 : t. 1, 28-29.

15 Article « Critique » (Médecine, t. V, $1792: 232$ ). 
naturelle des menstrues - même s'ils qualifiaient parfois de critique le temps nécessaire au processus. C'est le cas dans le traité de référence en matière de maladies des femmes, la somme de Jean Astruc (16841766 ${ }^{16}$. Les travaux contemporains sur les durées inégales de la vie humaine ont probablement favorisé le changement qui s'opère ensuite, comme l'illustre l'échelle de La Métherie évoquée ci-dessus. Quoiqu'elles restent en nombre limité dans les corpus interrogés, les occurrences des locutions contenant «critique» commencent à être plus fréquentes dans les années $1770^{17}$. Le témoignage de SamuelAuguste Tissot (1728-1797) en atteste. Le célèbre praticien suisse constate dès 1779 qu'«âge critique» sert - improprement - à dénommer ce changement physiologique propre aux femmes ${ }^{18}$. Cette appellation avait ainsi cours dans la langue courante sans être vraiment admise dans le vocabulaire savant, puisqu'elle est encore rare dans les imprimés médicaux. Son absence, il est vrai, traduit aussi le faible intérêt des médecins pour cette période de l'existence féminine. L'essor des écrits spécialisés va être propice à une démultiplication de locutions synonymes. L'actualisation, en 1799, de la brochure de Fothergill - Conseils aux femmes de quarante-cinq à cinquante ans (1788) - l'illustre clairement. Cette «période de la vie» féminine, dépourvue de dénomination qualifiante dans le texte originel, est désormais présentée comme l'«Époque critique propre à toutes les femmes » ${ }^{19}$. Ces expressions semblent donc faire vraiment souche dans les années 1800 et la forme « âge critique » l'emporte alors sur les autres.

Dans un tel contexte, comment fut reçue l'innovation de Gardanne? Nous pouvons ici nous appuyer sur les dictionnaires médicaux comme révélateurs des usages savants. "Ménespausie » est d'emblée introduit dans celui en cours dans les années 1810 et employé

\footnotetext{
16 Astruc 1761 : t. 2, 299-338.

17 Nous ne disposons d'aucun corpus adéquat pour mener une enquête quantitative solide. Nous nous fondons ici sur des recherches d'occurrences effectuées dans Google books [https://books.google.fr/] et surtout Gallica (BNF) [http://gallica.bnf.fr/], plus fiable, et qui comprend un nombre notable de textes médicaux numérisés.

18 Tissot $1779: 103$.

19 Fothergill an VIII : 11.
} 
dès 1818 dans une thèse soutenue à Paris ${ }^{20}$. Le Dictionnaire de médecine, dix ans plus tard, la conserve et signale l'existence de la forme «ménopause ». Mais l'entrée disparaît de la seconde édition, les termes sont même absents à Menstruation, long article qui dénonce pourtant le recours aux dénominations vulgaires ${ }^{21}$. Le mot savant rencontre donc des résistances durables, dont le Dictionnaire de médecine et de thérapentique médicale et chirurgicale (1877) rend parfaitement compte. L'entrée "Ménopause ou âge critique » comporte une vingtaine de lignes et s'achève sur un renvoi à "Âge critique », fort lui d'une soixantaine de lignes, pour décrire les «maladies de la ménopause $» . .{ }^{22}$. Ces évolutions complexes mettent au jour des tensions au sein du corps médical qui nous invitent à reconsidérer sous cet angle la production et la réception des premiers écrits spécialisés.

\section{La naissance d'une littérature médicale spécialisée}

Dans son Traité des nerfs et de leurs maladies (1779), Tissot met l'accent sur l'influence que les règles exercent sur ceux des femmes, en particulier à l'époque de leur cessation naturelle et déplore alors les carences d'un savoir médical incapable de les soulager. Jusqu'au mémoire de l'anglais Fothergill (1776), « on n'avoit rien de bon sur cette matière », or il est très peu répandu ${ }^{23}$. Ce vide de connaissance attire désormais l'attention. On voit ainsi le Journal des sçavans s'emparer d'une question - en latin - discutée à la Faculté de médecine de Paris, le 9 février 1786, et rédigée par Pierre-Joseph Morisot-Deslandes (1716-1792), président du jury d'un des grades passés par Claude-Bartholomé-Jean Leclerc :

Le traitement des maladies qui surviennent aux femmes lors de la cessation de leurs règles, quoique très difficile en lui-même, ne réussit-il pas mieux quand il est dirigé par la médecine rationnelle ?24.

\footnotetext{
20 Dictionnaire des sciences médicales: vol. Méd-Més (1819), 362 ; G. Maladière-Montécot, Considérations générales sur la ménespausie, cité par Rozenbaum \& Peumery 1990 : 70-71.

21 Adelon 1821-1828 : t. 14 (1826), 150-151; Adelon 1832-1844 : t. 19 (1839), 440-480.

22 Bouchut \& Després $1877: 33$ et 909.

23 Tissot $1779: 99$.

24 Journal des sçavans, nov. 1786 : 738-744 ; Leclerc 1786.
} 
L'abbé Tessier - le recenseur - estime que si ces maladies ont peu mobilisé les médecins jusque-là, contrairement à celles de la puberté ou des couches, la raison en est la plus grande difficulté à les connaitre, et non un motif d'indifférence. Il note que Morisot-Deslandes assigne à ces maux deux causes principales : le commencement de la vieillesse - auquel l'autre sexe est également soumis - et la pléthore, source la plus puissante de « ravages » sur le sexe féminin. Or, la conjugaison des deux causes, élément essentiel pour Morisot-Deslandes, va ensuite s'estomper dans l'appréhension d'un phénomène de plus en plus pensé comme une forme spécifique de vieillissement.

Les Conseils aux femmes de quarante ans (1787) paraissent ainsi dans un climat favorable. Ils émanent d'un autre docteur régent de la Faculté de médecine de Paris, Jean-Baptiste-Claude Jeannet des Longrois, dont une des thèses portait sur les hémorragies utérines ${ }^{25}$. La rédaction des Conseils répond à une ambition à la fois scientifique et mondaine d'un jeune docteur, qui voit dans la femme un être gouverné par sa sensibilité que la nature est impuissante à guérir, les "complications de l'âme» s'ajoutant aux causes physiques du moindre mal qui la touche et l'époque de la cessation des menstrues est celle « où la Nature opprimée a besoin plus que jamais d'appeler l'Art à son secours » ${ }^{26}$. Si la Gazette de santé se montre assez indulgente ${ }^{27}$, Jeannet de Longrois essuie une critique autrement plus virulente dans les Nouvelles on Annales de médecine, chirurgie et pharmacie et dont la portée générale mérite attention. Noël Retz (17..-1810), médecin ordinaire du roi, attaque tant le fond que la forme d'un livre dont le titre est des plus discordants avec son contenu :

il n'est pas plus question [...] des maladies des femmes de quarante ans, que de celles de tous les âges et des deux sexes; que les maladies dont il $\mathrm{y}$ est question sont décrites d'une manière très superficielle, beaucoup moins bien que dans le plus léger dictionnaire; que les remèdes conseillés pour les combattre, sont le plus souvent très-mal choisis $[\ldots]^{28}$

\footnotetext{
25 Jeannet des Longrois 1778.

26 Jeannet des Longrois $1787: 1-2$ et 8.

27 Gazette de santé, $1787, \mathrm{n}^{\circ} 45: 177-178$.

28 Retz 1789: 356-357; Rezt lui reproche aussi d'assimiler les femmes ménopausées à des « reines détrônées ».
} 
Le quatrième chapitre - de loin le plus conséquent - détaillait en effet les maladies "causées par le refoulement des règles", sans vraiment spécifier celles dues à leur disparition naturelle. Retz se gausse d'y trouver les pâles couleurs, propres aux jeunes filles et l'hypochondrie, un mal exclusivement masculin selon lui... Un différend majeur l'oppose à son confrère : il est partisan convaincu de laisser faire la Nature, sauf cas exceptionnels, seul conseil qu'un médecin puisse prodiguer à ses patientes.

La traduction en français de Fothergill, un savant de réputation européenne, apparaît clairement comme une réplique aux prescriptions mal avisées de Jeannet des Longrois ${ }^{29}$. Elle est d'ailleurs imprimée en 1788 sous le titre de Conseils pour les femmes de quarante-cinq à cinquante ans - absent de l'original - ou Conduite à tenir lors de la cessation des règles. La volonté de concurrencer Jeannet des Longrois sur le même terrain - en repoussant en outre de 5 ans l'âge d'entrée dans la période critique - conduit à soumettre au public éclairé des observations que le président de la Société médicale de Londres, John Fothergill (1712-1780), avait rassemblées en vue d'instruire ses pairs, en particulier ses jeunes confrères, ignorant où trouver des connaissances utiles sur le sujet ${ }^{30}$. Imprimée en 1776 dans les recueils de la Société, la dissertation ne fit jamais l'objet outre-Manche d'une diffusion comparable à celle qu'elle connut en France. Son principal objectif était de battre en brèche toutes les conceptions dévoyées, d'origine populaire ou savante, existant sur la ménopause :

Les opinions $[\ldots]$ plus ou moins erronées sur la cessation du flux menstruel, et ses suites, propagées d'âge en âge, ont rendu bien amers les momens de réflexion de beaucoup de femmes sensibles. Elles n'ont point été les seules qui [...] se soient laissées entrainer dans l'erreur ; car plusieurs de ceux même qui, par état, auraient dû s'en laisser moins imposer, ont été comme elles maîtrisés par les préjugés [...]. Mon dessein ici est de ramener le calme, d'éloigner toutes les appréhensions mal fondées que le sexe pourrait avoir, et de les remplacer par la sécurité qu'une confiance raisonnée peut donner, en sorte qu'avec peu de moyens, la nature puisse se suffire à elle-même en cette circonstance ${ }^{31}$.

29 Retz $1789: 363$

30 Fothergill 1776, $1788:$ 7. Pour sa biographie : Vicq d'Azyr 1785.

31 Fothergill $1788: 6$. 
Fothergill insiste d'abord sur la nécessité d'instruire les femmes (et les hommes) sur l'absence de malignité du sang évacué lors des menstruations. Le flux menstruel cesse naturellement une fois devenu inutile, conformément à la physiologie féminine et nombre de femmes n'en ressentent aucun effet notable sur leur santé. Il n'en nie pas pour autant que d'autres sont sujettes à des maux qui requièrent les secours de l'art, et en distingue essentiellement deux grandes catégories, ceux associés à la pléthore et les écoulements hémorragiques ${ }^{32}$.

Ce discours rassurant lui vaut des recensions élogieuses, notamment dans la Gazette de santé33, qui précise le nom du traducteur, Philippe Petit-Radel (1749-1815), ancien chirurgien militaire et docteur régent de la Faculté de médecine de Paris depuis $1782^{34}$. Ce dernier s'est vu confier la partie Chirurgie de l'Encyclopédie méthodique et a découvert le texte de Fothergill lors de ses dépouillements. Après un exil de plusieurs années sous la Révolution, Petit-Radel décide de rééditer la brochure, épuisée de longue date, avec cette fois une introduction et des notes. Or, sans retoucher le discours du médecin anglais, il en infléchit la portée : ses interventions, en particulier la préface, ont une tonalité alarmiste et moralisatrice. Il insiste sur la responsabilité des femmes dans les maux qui les frappent, sanctions de leur inconduite passée, et que certaines conservent l'âge venant. Ce moralisme s'enracine dans un bilan amer de la Révolution: la "régénération récente dans l'ordre actuel des choses est loin d'avoir amené un changement en mieux dans les mœurs $»^{35}$. Petit-Radel y déchiffre la menace d'une dégénérescence de la population. Aussi promet-il aux femmes très fécondes, des chances accrues de ménopause sans risque, alors que Fothergill ne tissait aucun lien entre maternité et dangerosité de la cessation des règles. Si les notes ont dans l'ensemble une teneur scientifique, en vue d'une mise à jour du contenu, certaines d'entre elles dramatisent à outrance les risques encourus. Petit-Radel s'y laisse aller à dévoiler l'ouverture d'un cadavre exhumant un vagin atrophié, sans égard pour ses lectrices, ou

\footnotetext{
32 Fothergill $1788: 10,12$.

33 Gazette de santé, 1788, n³4:133-134.

34 Pour sa biographie, Huguet 1991 : 362-363.

35 Fothergill an VIII : 8.
} 
encore diagnostique un nouveau mal inquiétant, les désirs sexuels hors de saison que certaines femmes peuvent ressentir au point de solliciter «les embrassemens de leur époux $»^{36}$. Ainsi, sous couvert de l'autorité de Fothergill, circule désormais un discours très ambivalent, réimprimé sans variante notable en 1812, signe qu'il continue à être une référence incontournable ${ }^{37}$.

Entre temps, plusieurs thèses ont été soutenues à la Faculté de médecine de Paris. Seul l'inventeur du mot «ménopause » transforme l'essai en un ouvrage de synthèse. Fils du chirurgien GardanneDuport (1746-1815), Charles-Pierre-Louis de Gardanne (1789-1827) appartient à une dynastie médicale déjà illustre sous l'Ancien régime ${ }^{38}$. Reçu docteur à la Faculté de médecine de Paris en juillet 1812, il s'installe peu après rue Notre-Dame des Victoires ${ }^{39}$ et s'il reste en marge du monde hospitalo-universitaire, Gardanne parvient néanmoins en une quinzaine d'années à prendre rang dans l'élite médicale parisienne. Outre les deux éditions de l'Avis aux femmes qui entrent dans l'âge critique (1816 et 1821), il poursuit des recherches publiées dans le Journal général de médecine, de chirurgie et de pharmacie et plus tard dans les Annales du Cercle médical de Paris, société dont il est membre et secrétaire de la commission des consultations en 182140. Gardanne s'est rapidement investi dans l'action sociale en devenant médecin de charité dans son arrondissement et fréquente le Paris mondain et philanthropique de l'époque, son beau-père, le sculpteur et graveur Nicolas Gatteaux (1751-1832), tenant salon dans les beaux quartiers. Il s'estime assez aguerri dès 1818 pour livrer des Réflexions philosophiques sur la médecine et le médecin. Un drame familial vient briser son ascension en 1824 et Gardanne meurt trois ans plus

36 Fothergill an VIII : 29 et 20. Ce "rebond de sexualité" figurait dans le traité général sur les maladies des femmes de Chambon de Montaux (1784) : Tillier 2005 : 5.

37 Voir aussi Giraudy an XIII, une autre traduction de Fothergill, conservée à peu d'exemplaires et que nous n'avons pas pu consulter.

38 La Gazette de santé signale sa parenté avec son fondateur, Jacques-Joseph Gardanne (1726-1786).

39 Gardanne 1812; nous avons mobilisé diverses sources pour reconstituer sa biographie, très sommaire dans les notices existantes dont AN, AJ 16/6755.

40 Annales du Cercle médical, 1820-1823 (3 vols). 
tard, dans des circonstances inconnues. Sa disparition ne suscite aucun écho ${ }^{41}$.

L'Avis aux femmes qui entrent dans l'âge critique fut pourtant un ouvrage abondamment commenté, en France et à l'étranger. C'est là ce qui nous importe ici, plutôt que de revenir sur son contenu luimême. Précisons seulement que l'auteur affiche deux objectifs, développer une branche négligée de la science médicale et effrayer un "sexe indifférent aux précautions à prendre", premier lectorat qu'il entend atteindre ${ }^{42}$. L'ouvrage se présente en trois parties - conservées en 1821 - physiologie de l'utérus à l'âge critique, règles d'hygiène et moyens préventifs, relevé des maladies et moyens curatifs, de loin la plus longue. Les recensions dont il fait l'objet sont riches d'enseignements. Elles nous dévoilent en particulier une divergence de points de vue dans le corps médical sur la cible éditoriale qui doit être visée ${ }^{43}$. Le London medical and physical journal et le Journal universel des sciences médicales considèrent que la ménopause et ses suites appellent avant tout un traitement scientifique utile aux hommes de l'art. Le langage sentimental qu'adopte Gardanne vient gâcher l'excellence du fond, selon le journal anglais, tandis que son confrère, plus sévère sur le contenu, juge son vocabulaire médical parfois désuet et profane. La Gazette de santé lui reproche au contraire de jargonner, et de lasser ses lectrices, avis que partage le Journal de médecine, chirurgie, pharmacie. Ce dernier pousse la critique au-delà : la plupart des médecins n'ont parlé du «temps critique des femmes » que dans des traités généraux, à juste titre, il n'y a là nullement matière à un livre savant. Gardanne aurait dû s'en tenir à l'hygiène, plus qu'à la thérapeutique. Le jeune médecin s'est livré à un exercice périlleux : prodiguer des conseils aux patientes et faire œuvre de science.

41 Sa femme Virginie meurt en 1824. Sur son mariage et sa descendance : Perrot \& Ribeill 1985 : 229-232; pour son décès, Naef $1978: 492$.

42 Gardanne 1816 : III-IV.

43 Les principales sont les suivantes : The London medical and physical journal, 1817 : 320-321. Journal de médecine, chirurgie, pharmacie, déc. 1816: 373-377 ; sep.-déc. 1821 : 339-340. Gazette de santé, 21 sept. 1816 : 214 ; 15 fév. 1822 : 37-39. Journal complémentaire du Dictionnaire des sciences médicales, déc. 1821: 154-157; Journal universel des sciences médicales, fév. 1822 : 170-186. 
Aussi une part notable des critiques portent-elles sur le contenu scientifique de l'ouvrage. L'hypothèse du «moteur séminal» pour expliquer la cause du flux menstruel est ainsi unanimement rejetée. Concernant l'hygiène, Gardanne tend à se perdre dans l'accessoire et les détails fastidieux - une «érudition de boudoir » selon la Gazette de santé - et, pour ces critiques les plus sévères, il rabâche des lieux communs. Qu'en est-il en termes de pathologie ? L'exactitude dans la description des maladies et des traitements est reconnue comme un des points forts de l'ouvrage. La nomenclature extensive des maux associés à la ménopause est à peine contestée. Seul le Journal universel des sciences médicales note que le tableau englobe parfois des maux prévalant à tous âges. Cet aspect ne fait désormais plus polémique et la réception de l'Avis aux femmes qui entrent dans l'âge critique témoigne bien de la montée en puissance d'une vision pathologique de la ménopause au cours de la période.

\section{L'hygiène de l'âge de retour des deux sexes}

Pour remettre en perspective les productions centrées sur l'âge critique des femmes, il est nécessaire de revenir sur l'attention croissante portée à une étape transitoire avant la vieillesse dans les échelles de la vie. La genèse de la pathologisation de la ménopause s'inscrit aussi dans l'émergence d'une médecine de l'«âge de retour» destinée aux deux sexes, en particulier dans les traités hygiénistes qui font florès à l'époque. La plus ou moins grande sexuation des conseils prodigués aux quarantenaires et aux cinquantenaires - et aux praticiens qui les soignent - est un indicateur très significatif pour apprécier l'incidence de la ménopause dans la perception d'un processus de vieillissement différencié et donne à voir au plus près la «fabrique» de l'asymétrie entre les deux sexes. Le premier traité dédié, du moins en partie, à la maturité déclinante paraît en 1836 et s'intitule précisément Le Médecin de l'âge de retour et de la vieillesse, ou Conseils aux personnes des deux sexes, qui ont passé l'âge de quarante-cinq ans (1836). Tandis que la vieillesse des unes et des autres fait l'objet d'un traitement indifférencié, l'âge de retour donne lieu à deux chapitres distincts.

Comme dans le cas de Gardanne, l'ouvrage correspond à un moment charnière dans la carrière de son auteur, Sébastien Guyétant 
(1777-1865) ${ }^{44}$. Fils de Jean-Frédéric, premier médecin de l'hôpital de Lons-le-Saulnier, il s'est formé à Paris où il est reçu docteur en 1801. Revenu dans sa ville natale, Guyétant devient médecin des épidémies de l'arrondissement et un pionnier de la pratique de la vaccine contre la variole - en 1836 il en revendique quelque 18000 - ce qui lui vaut nombre de distinctions. Par ailleurs secrétaire de la Société d'émulation du Jura, il s'intéresse à la botanique et à l'agriculture ${ }^{45}$ et appartient à un réseau important de sociétés savantes. Enfin au mitemps de sa carrière, vient la consécration avec son élection, le 5 avril 1825, à l'Académie royale de médecine.

Guyétant s'installe à Paris dix ans plus tard - il approche alors la soixantaine - lors de la publication du Médecin de l'âge de retour et de la vieillesse et ouvre un cabinet rue Taranne. Il s'attache désormais à diffuser des connaissances médicales élémentaires auprès de publics plus ou moins ciblés. Le Médecin de l'âge de retour et de la vieillesse s'adresse avant tout à la bonne société du temps, à même de le lire et d'en suivre les préceptes, et il connaît un succès très notable. Une seconde édition paraît dès 1837, une troisième, augmentée, en 1844, chez les frères Lagny. Il est traduit en allemand (1837) et en italien (1839) ${ }^{46}$. La Gazette médicale de Paris en donne une recension élogieuse dès le 13 février 1836, soulignant que le livre vient combler un manque : alors que les «trainers for health» de Londres en ont publié de qualité dans le genre, rien d'analogue n'existe en France ${ }^{47}$. L'ouvrage de Guyétant répond à deux exigences: simplicité et élégance dans l'expression, et rigueur du contenu scientifique qui s'appuie sur les recherches récentes des chimistes et des statisticiens. La Gazette médicale trouve uniquement à redire sur le titre choisi, peu à même à ses yeux de rallier un lectorat féminin...

À la suite de Hallé, Guyétant situe l'âge de retour entre 45-50 ans et 60-63 ans. La différenciation entre les deux sexes se cristallise

44 Pour sa biographie : Lachaise 1845 : 353-354; Thiébaud 1992 : 231 ; Blémont 1986 : 398.

45 Guyétant 1822.

46 Guyétant reçoit la légion d'honneur en 1837 et le dossier fait état du succès de l'ouvrage : AN, LH/1251/65.

47 Gazette médicale de Paris, 13 février 1836, nº $7: 112$. 
autour de cinq éléments ${ }^{48}$. Le premier d'entre eux est la temporalité du processus de vieillissement :

Après avoir brillé de tout l'éclat de la jeunesse et de la beauté, la femme arrive, ainsi que l'homme, mais un peu plus tôt que lui, à l'époque du déclin. C'est ordinairement, dans nos contrées, entre quarante-cinq et cinquante ans que les premiers signes de l'âge de retour se manifestent, chez elles, par la diminution et l'irrégularité de l'hémorragie périodique, dont la première apparition lui avait ouvert la voie de la fécondité, et dont la suppression définitive lui enlève la faculté de se reproduire et la réduit à son existence individuelle ${ }^{49}$.

Le « retour d'âge » fait basculer les femmes plus précocement dans l'âge de retour et change radicalement le sens de son existence. Cependant, une fois franchi ce cap critique, le temps suspend son vol pour nombre d'entre elles: «débarrassées définitivement de leur tribut menstruel», leurs forces redoublent. Ce regain de vitalité se traduit physiquement : elles ont le teint rosé, simulant la jeunesse, un agréable embonpoint, et recouvrent ainsi une partie de leurs attraits ${ }^{50}$. Le temps les rattrape entre 55 ans et 60 ans, leur apparence subit alors les signes $\mathrm{du}$ vieillissement communs aux deux sexes (canitie, chevelure clairsemée) et d'autres propres au vieillir au féminin (rides, changement de carnation, relâchement de la peau). Les hommes, quant à eux, voient apparaitre les premières marques de l'âge entre 45 ans et 50 ans, elles s'accentuent au fil des années, dont l'embonpoint, signe négatif ici et emblématique du vieillissement masculin. Le temps les affecte graduellement. Si leur corps n'est pas soumis à une « révolution» aussi sensible que celui des femmes, l'âge de retour les prive aussi des "mouvements critiques» et des évacuations salutaires fréquentes dans les jeunes organismes ${ }^{51}$. Le retour de l'âge entraîne une tendance à la pléthore, au masculin comme au féminin. La principale différence entre les deux sexes réside donc dans le caractère discontinu, propre aux femmes, des changements biologiques, alors qu'un processus progressif, plus insensible et moins visible, affecte les hommes.

\footnotetext{
48 Guyétant 1836 : 36-67(«De l'âge de retour chez l’homme, et des précautions qu'il demande ») et 68-107 («De l'âge de retour chez la femme, et des soins qu'il exige »).

49 Ibid. : 68.

50 Ibid. : 74-75.

51 Ibid. : 53.
} 
L'âge de retour correspond en second lieu à une époque de perfectionnement moral. C'est un des points saillants du discours de Guyétant. Chez l'homme, ce perfectionnement s'enracine dans un sentiment nouveau, la prévoyance, qui nait du besoin croissant de conservation que la nature lui fait ressentir. Il est conduit à dominer ses passions pour préserver ses forces, en particulier dans sa vie sexuelle : le "besoin de la reproduction cesse d'être impérieux chez la plupart des hommes $»^{52}$ qui savent désormais se satisfaire des «douces affections » familiales. À l'égard de la femme, la nature agit de même, mais en termes de compensation plus que de conservation. C'est la "perte de ses attraits » qui contribue à son perfectionnement moral et au "nouveau zèle» qui la rapproche de son mari et de ses enfants. La femme peut alors conserver - ou acquérir - un rôle éminent au sein d'une société « choisie ». Le repli salutaire vers la sphère domestique à l'âge de retour est donc commun aux deux sexes, mais il y a une différenciation sensible en amont sur les ressorts moraux qui le favorisent.

En troisième lieu, comme tout médecin hygiéniste, Guyétant s'attache à discerner les conduites qui exposent le plus les individus à une détérioration de leur santé et, dans leur mise au jour, les représentations sexuées s'avèrent être encore plus prégnantes. L'âge de retour est un moment critique pour les hommes qui ont vécu sans retenue, leurs excès de table et de chair leur font alors encourir des risques comparables à ceux des femmes au même âge. Le ton se durcit à l'égard de l'autre sexe. Le médecin parle alors « des abus et des écarts de régime ", des "passions déréglées », surtout "de graves infractions aux lois de la nature ». Sans stigmatiser ces comportements déviants, l'auteur reprend à son compte la relation forgée entre gravité des maux de la ménopause et degré d'accomplissement du destin biologique (mariage, maternité, allaitement) ${ }^{53}$. Guyétant tend ainsi aux deux sexes un miroir convenu: l'homme victime de ses excès nuit surtout à luimême, la femme transgressive perturbe aussi l'ordre social fondé sur les lois de la nature.

Aussi l'asymétrie entre les deux sexes reste forte en matière de recommandations: l'âge de retour demande aux hommes des

\footnotetext{
52 Guyétant $1836: 87$.

53 Ibid. : 72-73. Il évoque aussi les accouchements laborieux.
} 
«précautions » alors qu'il exige des « soins » pour les femmes. L'homme doit avant tout s'observer lui-même, c'est le moment de déterminer les maladies auxquelles son tempérament le prédispose. Les femmes, elles, ont besoin des secours de l'art. Au fil du chapitre, il y a d'ailleurs un glissement sensible, Guyétant s'adressant tout autant aux femmes confrontées à la ménopause qu’à ses confrères. Cette médicalisation est cependant dénuée de toute dramatisation. Au contraire, l'auteur entend rassurer - chiffres de mortalité à l'appui - et réduire à l'essentiel le spectre des maux courants (troubles digestifs, bouffées de chaleur, palpitations, sommeil agité, névralgies variées) : "Non», affirme-t-il, «l'âge critique n'est point le signal de cette effrayante invasion de maladie que rêve sans cesse l'imagination troublée d'un grand nombre de femmes ${ }^{54}$. S'il occulte ici la responsabilité de ses pairs, notons que Guyétant, certainement à dessein, élude le mot « ménopause » alors qu’il connaît parfaitement les travaux de Gardanne.

Enfin l'âge de retour doit signifier un changement de mode de vie, quel que soit le sexe. Sobriété alimentaire et exercices physiques adaptés à chacun sont les grands préceptes à respecter, la femme devant s'y plier plus tôt ${ }^{55}$. Au-delà de ces réformes attendues, Guyétant préconise des modifications plus radicales. Il suggère aux hommes de travailler moins, de se détourner des métiers dangereux pour en embrasser de plus sains, exploitant agricole étant le plus adapté et il espère être entendu du plus grand nombre... Les conseils aux femmes s'adressent sans détour à celles des classes nanties : elles doivent renoncer «aux plaisirs bruyants du monde, aux assemblées nombreuses, aux veilles prolongées, surtout si la fureur du jeu en aggrave encore les effets désastreux sur la santé $»^{56}$. Cet abandon de la vie mondaine va de pair avec des normes vestimentaires propres à cet âge qui se déclinent exclusivement au féminin (port de vêtements plus lâches, aucun décolleté). L'homme, convié lui-aussi à se retirer au sein de sa famille, conserve ainsi une marge de liberté au dehors, dont les femmes seraient privées pour préserver leur santé alors qu'au siècle

54 Guyétant $1836: 72$.

55 Ibid. : 77-78.

56 Ibid. : 83. 
précédent la «perte des fleurs» pouvait aussi être envisagée comme une libération et une source de mieux-être en société ${ }^{57}$.

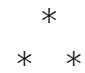

Le traité publié par Guyétant en 1822 fait apparaitre les évolutions à l'œuvre dans les prémices du processus de médicalisation de la «cessation des règles». L'invention de la ménopause prend racine avec l'émergence d'un nouvel âge de la vie dans les échelles médicales et une sexuation de ces échelles, au tournant des deux siècles, qui fait des facultés reproductrices le principal marqueur d'une différence entre les sexes dans les temporalités de la vie humaine. Soumis aux effets du "retour d'âge», le corps humain subit des altérations susceptibles d'affecter sa santé et de la compromettre dans la vieillesse. Cette période-charnière, ce moment critique, sonne l'heure $\mathrm{du}$ bilan et doit être l'occasion d'un changement salutaire dans le mode de vie. Elle nécessite aux yeux du médecin hygiéniste une réforme des conduites individuelles qui se décline, comme nous l'avons vu, selon les normes sexuées de la France postrévolutionnaire. L'une d'entre elles nous intéresse plus particulièrement ici : le corps féminin appelle une surveillance médicale plus étroite que le corps masculin et l'accompagnement médical recommandé pour faire face à la «cessation des règles » vient désormais étendre et renforcer cette asymétrie entre les sexes. Cette médicalisation du retour d'âge féminin, dans les discours, s'opère à l'époque, notons-le, sans pour autant forcément lui donner un caractère pathologique avéré et en dramatiser à outrance les dangers.

57 McClive 2010 : 297. 


\section{Bibliographie}

ADELON Nicolas-Philibert et al., 1821-1828, Dictionnaire de médecine, 21 vol., Paris, Béchet jeune.

—, 1832-1846, Dictionnaire de médecine ou répertoire général des sciences médicales .... Deuxième édition..., 30 vol., Paris, Béchet jeune.

ARIÈs Philippe, 1973, L'Enfant et la vie familiale sous l'Ancien régime, Paris, Le Seuil.

ASTRUC Jean, 1761[-1765], Traité sur les maladies des femmes avec un catalogue chronologique des médecins qui ont écrit sur ces maladies, 6 vol., Paris, Cavelier.

Beaugrand Émile, 1865, «Âge», in Amédée Dechambre (dir.), Dictionnaire encyclopédique des sciences médicales, t. II, Paris, G. Masson, P. Asselin, p. 135-145.

BlÉmont H., 1986, « Guyétant Sébastien », in Michel PrevosT et al. (dir.), Dictionnaire de biographie française, Paris, Le Touzey et Ané, fasc. XCVIII, p. 398.

Bouchut Eugène \& Armand Després, 1877, Dictionnaire de médecine et de thérapeutique médicale et chirurgicale... Troisième édition très augmentée, Paris, Germer-Baillière.

Bourdelais Patrice, 1993, L'Âge de la vieillesse, Paris, O. Jacob.

Daignan Guillaume, 1786, Tableau des variétés de la vie humaine, Paris, chez l'auteur.

DAubenton Louis-Jean-Marie, 1782, "Histoire naturelle de l'homme », in Encyclopédie méthodique. Histoire naturelle des animaux, Paris, Panckoucke, p. LX-LXXVI.

—, an VI, Mémoire sur les indigestions qui commencent à être plus fréquentes, pour la plupart des hommes, à l'âge de quarante ou quarante-cinq ans..., Paris, Huzard et Villiers.

DeLANOË Daniel, 2006, Sexe, croyance et ménopause, Paris, Hachette.

Dictionnaire de l'Académie française, éditions de 1694, 1762, 1798, 1835, 1932-1935: Dictionnaires d'autrefois, ARTFL Project, Université de Chicago.

Dictionnaire des sciences médicales, 1812-1822 (58 vols), Paris, Panckoucke.

Encyclopédie méthodique, Médecine, 1787-1830 (14 vols), Félix VICQ D’AzYR (éd.), [continué par] Jacques Louis MOREAU DE LA SARTHE (éd.), Paris, Panckoucke/Agasse.

FODÉRÉ François-Emmanuel, 1813, Traité de médecine légale et d'bygiène publique ou de police de santé..., Paris, Mane.

FOTHERGILL John, 1776, "On the management proper at the cessation of the memses », Medical observations and inquiries, t. V, Londres, Cadell, p. 160-186.

-, 1788, Conseils pour les femmes de quarante-cinq à cinquante ans, ou conduite à tenir lors de la cessation des règles..., Londres et Paris, Briand. 
—, an VIII [1799-1800]. Conseils aux femmes de quarante-cinq à cinquante ans... Traduit ..., et augmenté de notes par le D. Petit-Radel. Seconde édition, Paris, Gabon.

—, 1812, Conseils aux femmes de quarante-cinq à cinquante ans... Troisième édition, Paris, Méquignon-Marvis.

Gardanne Charles-Pierre-Louis de, 1812, «Dissertation sur les avis à donner aux femmes qui entrent dans l'âge critique », Paris, thèse de la Faculté de médecine.

—, 1816, Avis aux femmes qui entrent dans l'âge critique, Paris, Gabon.

—, 1818, Réflexions philosophiques sur la médecine et le médecin, Paris, Chanson.

—, 1821, De la ménopause ou de l'âge critique des femmes, Paris, Méquignon-Marvis.

GIRAUDY Charles-François-Simon, an XIII [1805], Conseils aux femmes sur les moyens de prévenir ou d'arrêter les suites fâcheuses de leur temps critique par le Docteur Fothergill de Londres, traduit de l'anglais... Paris, Crochard.

GUYÉTANT Sébastien, 1822, Essai sur l'état actuel de l'agriculture dans le Jura, les améliorations qu'elle a reçues depuis 30 ans, et celles dont elle paroît encore susceptible, Lonsle-Saulnier, Gauthier.

—, 1836, Le Médecin de l'âge de retour et de la vieillesse, Paris, l'auteur et Dufey.

Hallé Jean-Noël, 1787, «Âge», Encyclopédie méthodique, Médecine, t. I, Paris, Panckoucke, p. 358-361.

Hoffmann Paul, 1995 [reprod. de l'édition de Strasbourg, 1977], La Femme dans la pensée des Lumières, Genève, Slatkine / Paris, Diff. Champion.

Huguet Françoise, 1991, Les Professeurs de la Faculté de médecine de Paris. Dictionnaire biographique 1794-1939, Paris, Institut national de recherché pédagogique et Éditions du CNRS.

JEANNET DES LongroIs Jean-Baptiste-Claude, 1778. Quaestio medica... An pertinaci et inveteratae uteri haemorrhagiae, vinum antiscorbuticum, Parisiis, Quillau.

—, 1787, Conseils aux femmes de quarante ans, Paris, Méquignon.

KNIBIEHLer Yvonne \& Catherine MARAND-Fouquet, 1983, La Femme et les médecins, Paris, Hachette.

[LACHAise Claude] 1845, Les Médecins de Paris jugés par leurs auvres..., Paris, chez l'auteur.

LA MÉTHerie Jean-Claude, 1804, Considérations sur les êtres organisés, Paris, H.L. Perronneau.

LE Bras Hervé, 2000, Naissance de la mortalité. L'origine politique de la statistique et de la démographie, Paris, Gallimard / Le Seuil, coll. « Hautes Études ».

LECLERC Claude-Bartholomé-Jean, 1786, An morborum, cessante mensium fluxu, therapeia, quae difficillema, rationali medicina duce, felicior? (Praes. Petro Josepho Morisot Deslandes. Cand. Claudio Bartholomaes Joanne Le Clerc), Paris, Quillau. 
LIOTARD-SCHNEIDER Frédérique, 2010, «Les experts de l'intime et les femmes, médecins et démographes en France de 1945 à 1975 », thèse d'histoire, Paris Ouest-Nanterre.

LIVI Jocelyn, 1984, Vapeurs de femmes, essai historique sur quelques fantasmes médicaux, Paris, Navarin.

MCClive, Cathy, 2010, «Quand les fleurs s'arrêtent. La ménopause et l'imaginaire médical aux XVII e et XVIII ${ }^{\mathrm{e}}$ siècles ", in Cathy MCClive \& Nicole PellegriN (dir.), Femmes en fleurs, femmes en corps: sang, santé et sexualités du moyen âge aux lumières, Saint-Étienne, Presses universitaires de Saint-Étienne, p. 277-299.

NAEF Hans, 1978, "Die Familie Gatteaux», in Die Bildniszeichnungen von J.-A.-D. Ingres, t. 2, Berne, Benteli, p. 485-502.

Perrot Michelle \& Georges Ribeill, 1985, Le Journal intime de Caroline B., Paris, Montalba.

PORRET Michel, 2010. "La médecine légale entre doctrine et pratique», Revue d'histoire des sciences bumaines, 1-22, p. 3-15.

Recueil des lois composant le Code civil..., premier volume, deuxième partie, Paris, Rondonneau, an XI.

ReTz Noël, 1789, Nonvelles ou Annales de médecine, chirurgie et pharmacie, t. V, Paris, Méquignon.

Rozenbaum Henri \& Jean-Jacques Peumery, 1990, Histoire illustrée de la ménopause de l'Antiquité à nos jours, Paris, R. Dacosta.

Roussel Pierre, 1775, Système physique et moral de la femme, Paris, Vincent.

STEWART Joan Hinde, 2010, The Enlightenment of Age: women, letters and growing old in eighteenth-century France, Oxford, Voltaire Foundation, coll. «Studies on Voltaire and the eighteenth century ».

ThiÉBAud Jean-Marie, 1992, Médecins et chirurgiens de Franche-Comté du Moyen Âge au Premier Empire, Peronnas, Éditions de la Tour Gile.

TiLlier Annick, 2005, «Un âge critique. La ménopause sous le regard des médecins des XVIII' et XIXe siècles », Clio. Histoire, femmes et sociétés, 21, p. 269-280.

Tissot Samuel-Auguste, 1779, Traité des nerfs et de leurs maladies..., t. II, part. I, Paris, Didot.

Troyansky David G., 1989, Old Age in the Old Regime. Image and Experience in Eighteenth Century France, Ithaca, Cornell University Press.

VANDENESSE Urbain de, 1751, «Âge », in Diderót \& D’Alembert (dir.), Encyclopédie on Dictionnaire raisonné..., t. I, Paris, Briasson, p. 170-171.

VICQ D’Azyr Félix, 1785, "Éloge de M. Fothergill», Histoire de la Société royale de médecine. Années 1780 et 1781, Paris, Barrois, p. 50-84. 
